\title{
Preparation of Ultrathin Nanowires Using Superfluid Helium Droplets
}

\author{
Elspeth Latimer, Daniel Spence, Cheng Feng, Adrian Boatwright, Andrew M. Ellis,* and Shengfu Yang* \\ Department of Chemistry, University of Leicester, Leicester LE1 7RH, United Kingdom \\ Supporting Information
}

\begin{abstract}
Direct preparation of long one-dimensional (1D) nanostructures with diameters $<10 \mathrm{~nm}$ inside superfluid helium droplets is reported. Unlike conventional chemical synthetic techniques, where stabilizers, templates, or external fields are often required to induce $1 \mathrm{D}$ growth, here, we exploit the use of quantized vortices to guide the formation of ultrathin nanowires. A variety of elements have been added to the droplets to demonstrate that this is a general phenomenon, including $\mathrm{Ni}, \mathrm{Cr}, \mathrm{Au}$, and $\mathrm{Si}$. Control of the length and diameter of the nanowires is also demonstrated.
\end{abstract}
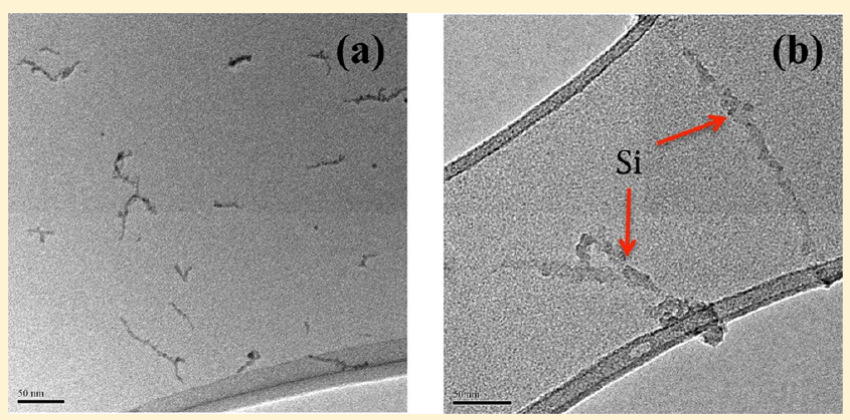

KEYWORDS: Helium droplet, vortex, nanoparticle, nanowire, transmission electron microscopy

$\mathrm{N}$ anostructures extended in one dimension (1D nanostructures), such as wires and tubes, have attracted much attention because of their potential applications in mesoscopic physics and in the fabrication of nanodevices. ${ }^{1-5}$ Ultrathin $1 \mathrm{D}$ nanostructures with diameters less than $10 \mathrm{~nm}$ possess unusual properties related to quantum confinement and the significantly increased surface-to-volume ratio, such as quantum conductance, ${ }^{6,7}$ ballistic conduction, ${ }^{8,9}$ ferromagnetism, ${ }^{10}$ negative magnetoresistance, ${ }^{11}$ and low thermal conductivity. ${ }^{12}$ Many exciting applications have already been envisaged from these properties including the development of chemical and biological sensors, ${ }^{13,14}$ catalysts, ${ }^{15}$ and components for miniature electronic circuits. ${ }^{16}$

Synthesis of ultrathin nanowires generally requires some source of anisotropy to bias the growth process in only one direction. To achieve this anisotropy, a variety of approaches have been used such as templates, ${ }^{17,18}$ ligand control, ${ }^{19}$ and oriented attachment ${ }^{20}$ (more information can be found in recent review articles by Xia et al., ${ }^{5}$ and Ozin et al., ${ }^{21}$ and references therein). In the presence of a weak binding stabilizer, ultrathin nanowires can now be synthesized even in aqueous solutions by reduction of inorganic metal precursors. ${ }^{22}$ Although most research in this field focuses on monometallic nanowires, 1D nanostructures composed of two different metals have also been reported, ${ }^{23-25}$ which could offer improved electronic, magnetic, and catalytic properties when compared with monometallic ones. Nevertheless, to date, all of the synthetic approaches are system-dependent and in only a few cases have ultrathin nanowires been produced without any stabilizer, template, or external field. ${ }^{26,27}$ For nanowires involving more than one type of material, simultaneous control of the composition and morphology remains a great challenge owing to the different nucleation and growth rates of different components.

In this study, we present an alternative method for constraining growth in one dimension. This approach uses superfluid helium droplets, which are large helium clusters with diameters ranging from a few nanometers to several micrometers. $^{28}$ The unique features derived from using helium droplets as the growth medium for the formation of nanoparticles include (1) a very low temperature $(0.38 \mathrm{~K})$, (2) an exceptionally high thermal conductivity (which derives from the fact that helium is superfluid at $0.38 \mathrm{~K}$ ), (3) the ability to add materials to the droplets sequentially, enabling the easy formation of core-shell nanoparticles, and (4) the chemical inertness of helium, which allows highly reactive materials to be incorporated into nanoparticles. These factors offer a route to a large array of new and potentially very interesting types of nanoparticles. ${ }^{29}$ Although still in its infancy, a number of metallic nanoparticles have been synthesized using this technique and have been removed from the helium droplets for transmission electron microscopy (TEM) investigations, including $\mathrm{Ag} / \mathrm{Au}$ nanoparticles composed of $\sim 500$ metal atoms, ${ }^{30}$ pure $\mathrm{Ag}$ nanoparticles composed of over $6000 \mathrm{Ag}$ atoms, ${ }^{31} \mathrm{Ag} / \mathrm{Au}$ and $\mathrm{Ni} / \mathrm{Au}$ core-shell nanoparticles, ${ }^{32}$ and polycrystalline Ag nanoparticles. ${ }^{33}$ To form $1 \mathrm{D}$ nanostructures in helium droplets, we will take advantage of another unique feature of superfluid helium, the presence of quantized vortices, which provide anisotropy that can guide the one-dimensional growth of particles.

Received: March 13, 2014

Revised: April 4, 2014

Published: April 17, 2014 

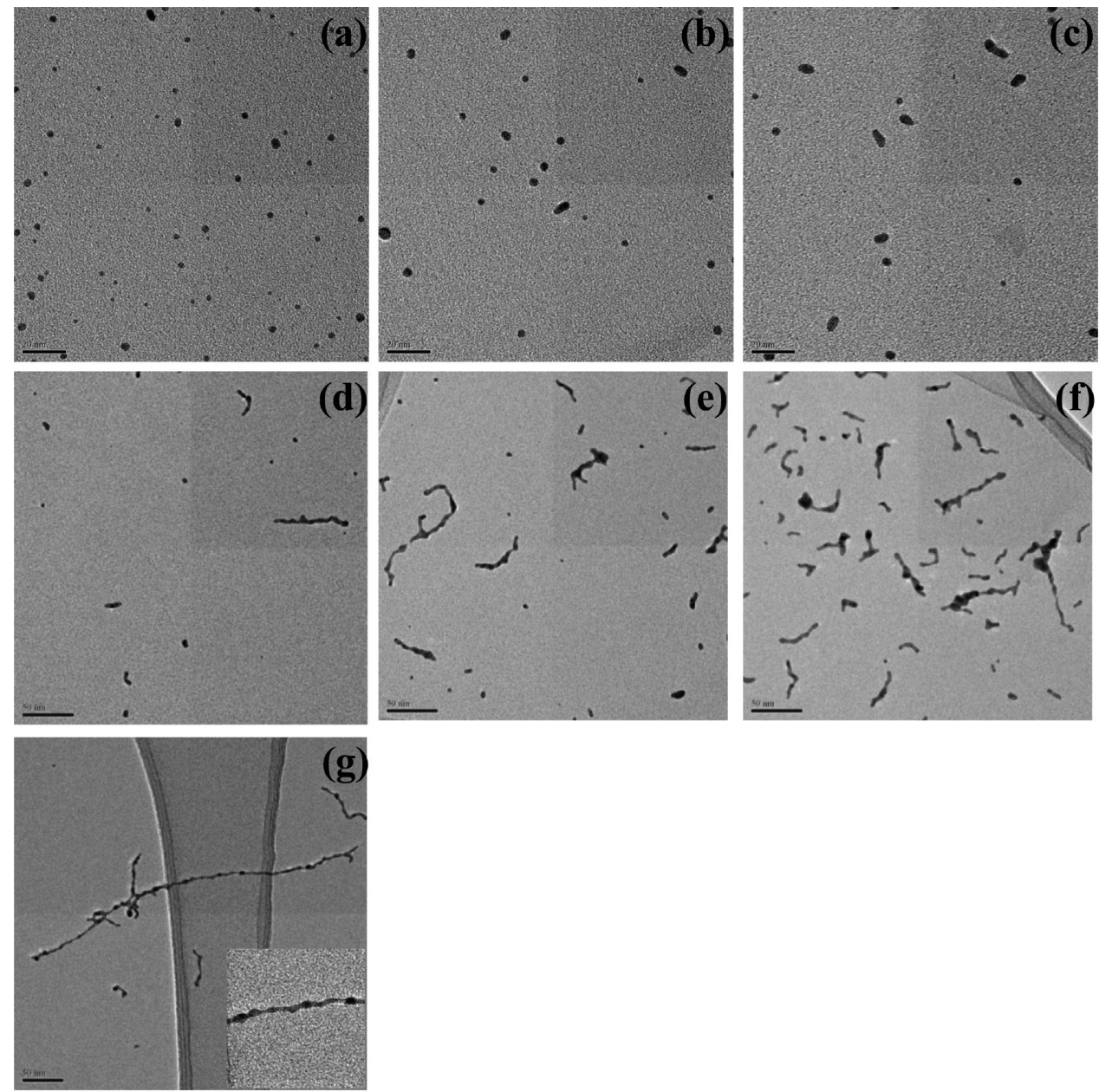

Figure 1. TEM images of Au nanostructures formed in helium droplets of different initial diameters: (a) $25 \mathrm{~nm},(\mathrm{~b}) 55 \mathrm{~nm},(\mathrm{c}) 75 \mathrm{~nm},(\mathrm{~d}) 135 \mathrm{~nm}$, (e) $250 \mathrm{~nm}$, (f) $790 \mathrm{~nm},(\mathrm{~g}) 1.7 \mu \mathrm{m}$. Note that (a)-(c) have a scale mark of $20 \mathrm{~nm}$, whereas for (d) $-(\mathrm{g})$, this is $50 \mathrm{~nm}$. For all of these experiments, the Au oven was kept at a fixed temperature of $1300 \mathrm{~K}$.

Quantized vortices are one of the most dramatic hallmarks of superfluidity and describe the behavior of the superfluid under rotation. ${ }^{34}$ The first direct images of quantized vortices in bulk superfluid helium were made relatively recently by passing $\mathrm{H}_{2}$ through superfluid helium, which then condensed into solid particles with sizes of the order of micrometers. ${ }^{35-38}$ In the superfluid phase, these particles align into long chains that can then be imaged using light scattering. The formation of chains was attributed to pinning of the particles to the quantized vortices by an attractive force, which derives from a weak pressure gradient induced by the circulation of the fluid around the vortex line. ${ }^{34}$ In a very different experiment, laser ablation of metals has been used to produce nanowires in bulk liquid helium. ${ }^{39}$ In the superfluid phase, very long filaments $(\sim 15$ $\mathrm{mm}$ ) composed of many entangled wires (thickness 2-10 nm) were produced, which were attributed to growth along quantized vortices.

The presence of quantized vortices in nanoscale helium droplets has been proposed on a number of occasions, ${ }^{40-42}$ but it is only very recently that experimental evidence has been obtained. Gomez et al. ${ }^{43}$ observed elongated track-shaped deposits when multiple $\mathrm{Ag}$ atoms were added to helium droplets of diameters larger than $300 \mathrm{~nm}$. The overall lengths of these were broadly consistent with the diameters of the helium droplets and provided evidence for the pinning of $\mathrm{Ag}$ atoms to quantized vortices extending across the helium droplets. A more recent study by Spence et al. ${ }^{44}$ followed an experimental procedure similar to that of Gomez et al., ${ }^{43}$ and through careful control of the doping conditions, both $\mathrm{Ag}$ nanoparticle arrays and nanorod chains were observed. These 1D Ag nanostructures had lengths up to several hundred nanometers and showed a uniform spacing between the particles and nanorods comprising the chain, mimicking the findings from the $\mathrm{H}_{2}$ experiments in bulk superfluid helium mentioned earlier. ${ }^{35-38}$ In particular, the formation of chains consisting of spherical nanoparticles demonstrated that the aligning force is derived from the helium droplet rather than any anisotropy in the particles.

Given the burgeoning evidence that now exists for quantized vortices in helium droplets, it is possible to consider utilizing these to form a variety of extended $1 \mathrm{D}$ nanostructures and the aim of this work is to demonstrate this capability. Specifically, we show that wires with diameters $<10 \mathrm{~nm}$ and extending for hundreds of $\mathrm{nm}$ can be formed for $\mathrm{Au}, \mathrm{Ni}, \mathrm{Cr}$, and $\mathrm{Si}$ when the helium droplet size becomes sufficiently large. These observations suggest that helium droplets have the potential to be used as a means of producing $1 \mathrm{D}$ nanostructures for a very wide range of materials.

We start by presenting our findings for gold. We investigated the progressive evolution of gold nanostructures grown in 
helium droplets of different mean diameters, $D$, ranging from $25 \mathrm{~nm}$ to $1.7 \mu \mathrm{m}$. ${ }^{45}$ These nanostructures were grown inside the helium droplets and then deposited on a solid surface by collision of the helium droplet beam with the surface. Figure 1 shows images from transmission electron microscopy (TEM) of the resulting surface deposits. With helium nanodroplets composed of $<10^{6}$ helium atoms $(D \approx 25 \mathrm{~nm})$ we see only spherical Au nanoparticles (see image a). For $D \approx 55 \mathrm{~nm}$, the TEM image in Figure $1 \mathrm{~b}$ shows both spherical Au particles and some elongated ones, but as the helium droplet size increases, the elongation becomes more pronounced (see Figure $1 \mathrm{c}-\mathrm{g}$ ). For helium droplets with a mean diameter of $135 \mathrm{~nm}(2.8 \times$ $10^{7}$ helium atoms), the nanowires have a length of $20 \pm 7 \mathrm{~nm}$ and a thickness of $3.9 \pm 0.5 \mathrm{~nm}$ on average (statistics derived from 100 nanowires in 15 TEM images). For $D \approx 1.7 \mu \mathrm{m}, \mathrm{Au}$ nanowires with length $>250 \mathrm{~nm}$ and a thickness of $3.6 \mathrm{~nm}$ were observed (see Figure $1 \mathrm{~g}$ for an example). Note that this is very different behavior from $\mathrm{Ag}$ in large helium droplets, where chains of particles with interparticle separations in excess of 10 nm were observed. ${ }^{44}$ In contrast, fully connected nanowires are observed when gold is added to large helium droplets.

In the expanded view shown in the bottom right of Figure 1g, a "peas-in-a-pod" structure is clearly seen, indicating that the formation of nanowires in the largest droplets studied probably involves the aggregation of smaller particles formed via a multicenter growth mechanism. Multicenter growth can occur in large helium droplets when the rate of addition of a dopant is faster than the transit time for the dopant to move from the surface to the center of the droplet. ${ }^{46}$ When this occurs, each "pea" is formed at some random nucleation site within the droplets and then eventually migrates to the vortex, where it becomes trapped. Presumably, additional Au atoms are able to reach the vortex lines and partly fill the gaps between these particles, creating the structure seen.

Some nanowires in Figure $1 \mathrm{~d}-\mathrm{g}$ show branches. In these images, the nanowires are relatively sparse, so the chance of one wire landing on top of another in the deposition process is very small. Consequently, the branched structure is likely to be intrinsic to nanowires formed in helium droplets. One possibility derives from multicenter growth because this leads to initial nucleation at different locations away from the vortices. If a nanoparticle formed at a location remote from the vortex approaches the vortex by diffusion and fails to reach either end of the nanowire, eventually it will encounter a continuous length of nanowire and attach to the side, starting a branch. This process is similar to the aggregation-limited aggregation occurring in wet-chemistry synthesis of nanomaterials, where diffusion is the primary means of particle transport. ${ }^{47,48}$ Another possibility is that more than one vortex may be present in some helium droplets. Vortex arrays are well known in bulk superfluid helium ${ }^{34}$ and are conceivable in helium droplets if the latter are formed with sufficient angular momentum. This will complicate the particle aggregation process and may lead to separate strands of nanowires being formed in a helium droplet, which may become entangled or even fuse when deposited onto a surface.

At a constant oven temperature the diameter of the nanowires decreases slightly as the helium droplet size increases, but the length of the nanowires increases accordingly. In another set of experiments, we investigated the dependence of the length and diameter of gold nanowires on the Au doping rate. Here, we kept the source temperature of helium droplets at $5 \mathrm{~K}$, corresponding to helium droplets with a diameter of 1.7 $\mu \mathrm{m}$, and then varied the partial pressure of gold vapor in the evaporator. As seen from Figure 2, the diameter of the

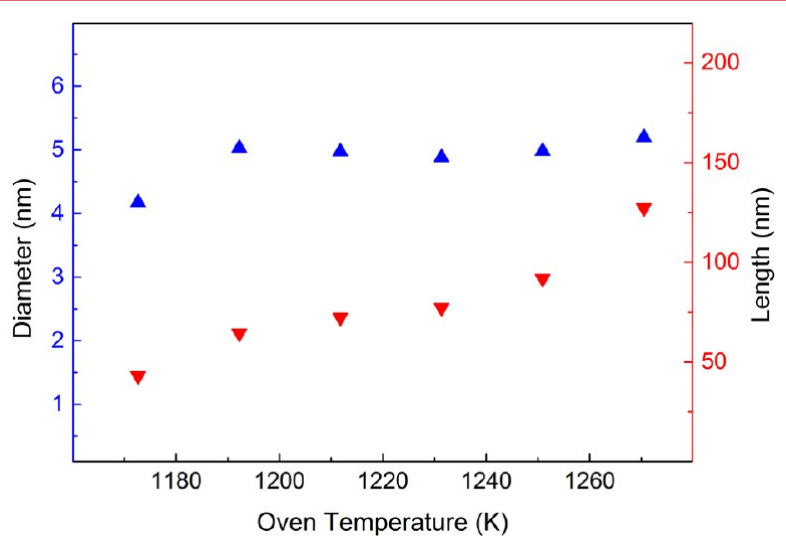

Figure 2. Dependence of the diameter (blue $\boldsymbol{\Delta}$ ) and the length (red $\boldsymbol{\nabla})$ of Au nanowires on the oven temperature for helium droplets with a mean initial diameter of $1.7 \mu \mathrm{m}$.

nanowires increases at lower oven temperatures but reaches a near constant value of $5 \mathrm{~nm}$ at higher doping rates. In contrast, the length of the nanowire increases monotonically with the oven temperature. This shows that by careful control of the doping rate and the size of helium droplets, the length of the nanowires can be controlled while maintaining a very narrow diameter.

We now wish to explore if continuous nanowire formation in helium droplets is the norm. To address this point we turn to other elements, specifically $\mathrm{Ni}, \mathrm{Cr}$ and $\mathrm{Si}$. Figure 3 shows TEM images of nickel nanoparticles grown in helium droplets. The behavior of $\mathrm{Ni}$ is similar to $\mathrm{Au}$. In Figure $3 \mathrm{a}$ and $\mathrm{b}$, we see a transition from spherical particles to nanorods and then the nanorods become progressively longer as the helium droplet size increases. For the largest helium droplets, $D \approx 1.7 \mu \mathrm{m}$, we have obtained continuous nanowires with lengths of a few hundred $\mathrm{nm}$ and a diameter of $4 \mathrm{~nm}$. In contrast to the other TEM images in Figure 3, where the deposition time was $30 \mathrm{~s}$, the image in Figure $3 \mathrm{f}$ shows Ni nanowires produced from a 5 min deposition process. Significant entangling of nanowires occurred because of this relatively long deposition time. At the lower-right corner of this image a straight wire with a length exceeding $300 \mathrm{~nm}$ is observed spanning a gap across the lacey carbon film. In addition, some branches are clearly seen on this long wire. Because the wires are unlikely to join together or land on top of each other in a hole on the lacey carbon substrate, this suggests that the branched structure is formed inside the helium droplet.

The progressive growth of $\mathrm{Cr}$ nanostructures with the diameter of the helium droplets was also investigated. Similar to $\mathrm{Au}$ and $\mathrm{Ni}$ spherical $\mathrm{Cr}$ nanoparticles were seen for small helium droplets but wirelike structures appeared for larger helium droplets. In the largest helium droplets employed in the Cr experiments, where $D \approx 790 \mathrm{~nm}$, Cr nanowires with a mean length of $69 \mathrm{~nm}$ and a diameter of $3.3 \mathrm{~nm}$ were observed (see Figure 4a).

We also attempted to form $1 \mathrm{D}$ nanostructures using a nonmetallic material, Si. The TEM images of Si nanowires have a relatively poor contrast compared to the metals described above because $\mathrm{Si}$ does not scatter electrons as readily. For helium droplets with $D \approx 1.7 \mu \mathrm{m}$, we observed Si nanowires 

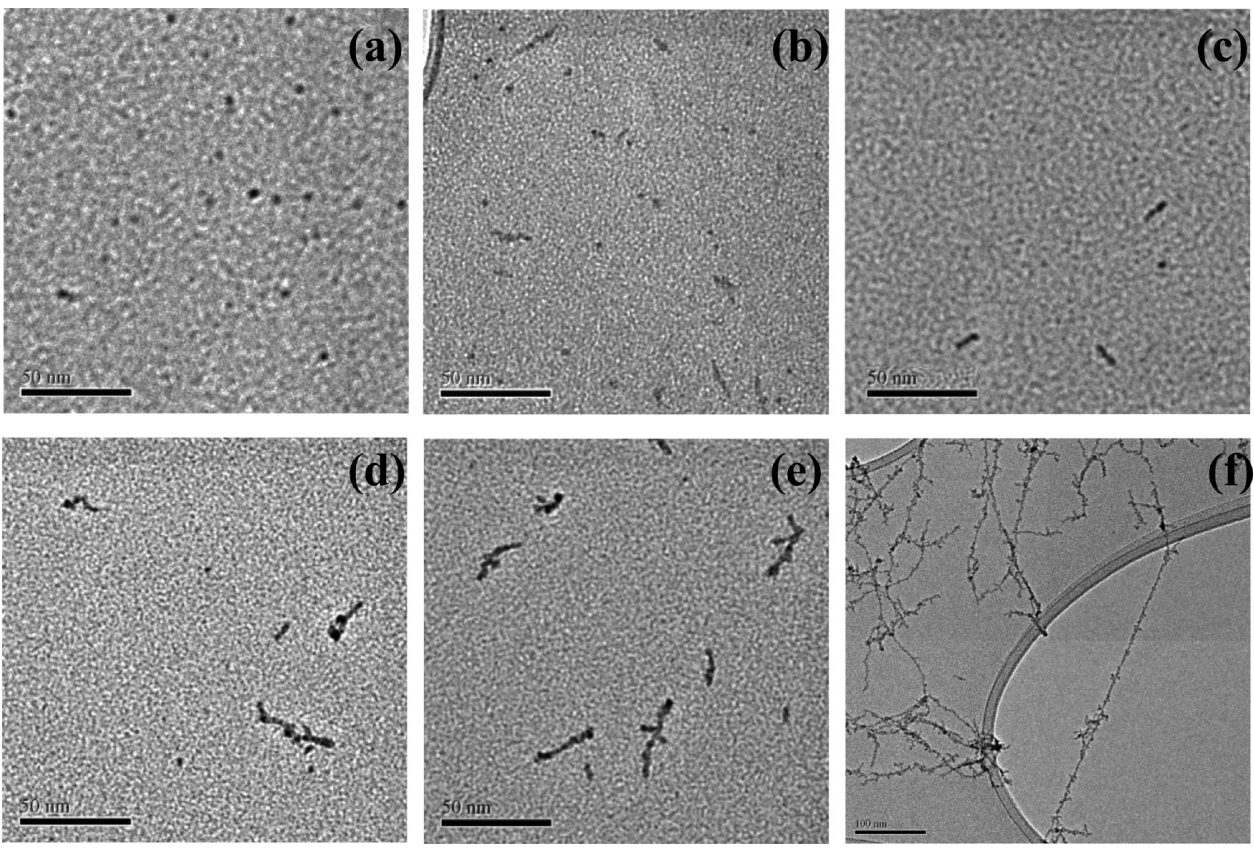

Figure 3. TEM images of Ni nanostructures formed in helium droplets with the following mean initial diameters: (a) $55 \mathrm{~nm}$, (b) $63 \mathrm{~nm}$, (c) $135 \mathrm{~nm}$, (d) $245 \mathrm{~nm}$, (e) $790 \mathrm{~nm}$, (f) $1.7 \mu \mathrm{m}$. Images (a)-(g) each have a scale mark of $50 \mathrm{~nm}$ while (f) has a scale mark of $100 \mathrm{~nm}$. For all of these experiments the $\mathrm{Ni}$ oven was kept at a fixed temperature of $1700 \mathrm{~K}$.
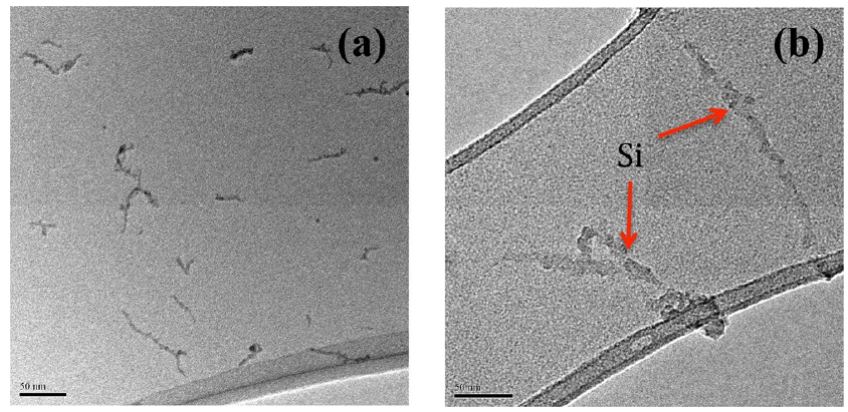

Figure 4. TEM images of (a) $\mathrm{Cr}$ and (b) Si nanowires formed in superfluid helium. For the chromium experiment the mean initial helium droplet size was $790 \mathrm{~nm}$ and for silicon it was $1.7 \mu \mathrm{m}$. The scale mark is $50 \mathrm{~nm}$ in both images. The oven temperatures were 1500 and $1400 \mathrm{~K}$ for $\mathrm{Cr}$ and $\mathrm{Si}$, respectively.

with lengths exceeding $100 \mathrm{~nm}$ (see Figure 4b). These nanowires are shorter than those obtained for $\mathrm{Ni}$ and $\mathrm{Au}$ because a lower vapor pressure was used in the Si oven.

In summary, this work shows that nanowires can be successfully grown in helium droplets for several different elements. Given these observations, our expectation is that the formation of nanowires in large helium droplets is a general phenomenon. Unlike other techniques, growth of the nanowires in helium droplets is spontaneous, requiring no extra guiding force other than that which is intrinsic to the droplets, quantized vortices. This, therefore, offers a route to prepare many different types of nanowires, including metallic and nonmetallic, and has the potential to become a generic method for the direct preparation of $1 \mathrm{D}$ nanostructures. Because of the high degree of control offered by superfluid helium droplets, it is possible to tailor the properties of nanowires for specific applications, such as sensors, catalysts, and photovoltaics.

Multilayer nanowires are also viable via this route. The traveling helium droplets can sequentially acquire different types of materials when they pass through different pickup regions, ${ }^{32}$ making it possible to form multilayer nanowires. We note that in recent experiments in our laboratory on the formation of $1 \mathrm{D} \mathrm{Ag}$ nanostructures, where Si was added to helium droplets after $\mathrm{Ag}, \mathrm{Ag}-\mathrm{Si}$ nanowires were formed with $\mathrm{Si}$ covering the surface of $\mathrm{Ag}$ nanorods and in-filling the gaps between the $\mathrm{Ag}$ nanorods. ${ }^{44}$ This layer-by-layer growth offers opportunities for synthesizing many multilayer nanowires, such as bimetallic and hybrid metal-semiconductor nanowires, where each material can be independently controlled. A special case of hybrid metal-semiconductor nanowires is the noble metal and semiconductor binary system, where the strong surface plasmon resonance of the noble metal components can significantly enhance the optical properties of the semiconductors through energy or carrier transfer. This combination could open up many applications in photonics, plasmonics, and photovoltaics.

\section{METHOD SUMMARY}

The apparatus for the synthesis of nanoparticles and nanowires in helium droplets has been detailed previously. ${ }^{29,32,44}$ Briefly, the vacuum system consists of four interconnected ultrahigh vacuum (UHV) regions: a source chamber, a dopant pick-up region, a deposition station, and a mass spectrometer. The helium droplets are produced in the source region by passing high purity helium (99.9999\%) under pressure through a $5 \mu \mathrm{m}$ nozzle that can be cooled to temperatures as low as $4 \mathrm{~K}$ by a closed-cycle helium cryostat (Sumitomo RDK-415D). For the work discussed here, a helium stagnation pressure of 15 bar was used in all experiments. On leaving the source chamber, the droplets were collimated into a beam by passage through a 0.5 mm skimmer.

Nanoparticles and nanowires were formed by addition of multiple atoms to the helium droplets from atomic vapor generated from a resistively heated oven whose temperature was measured using a thermocouple. Further downstream is a 
deposition station coupled with a load-lock chamber, which allows the transportation of deposition targets to external microscopy facilities without interrupting the vacuum in the rest of the apparatus. In all of the experiments, nanoparticles were deposited onto lacey carbon substrates for $30 \mathrm{~s}$, except that the $\mathrm{Ni}$ nanowires formed in helium droplets with a diameter of $1.7 \mu \mathrm{m}$ were deposited for $5 \mathrm{~min}$ (see Figure 3f). TEM imaging was carried out using a JEOL JEM-2100 LaB6 transmission electron microscope (see Supporting Information for results).

\section{ASSOCIATED CONTENT}

\section{S Supporting Information}

TEM image of Ni nanowires. This material is available free of charge via the Internet at http://pubs.acs.org.

\section{AUTHOR INFORMATION}

\section{Corresponding Authors}

*S. Yang. E-mail: sfy1@le.ac.uk. Phone: +44-116 2522127. Fax: +44-116 2523789.

*A. M. Ellis. E-mail: ame2@le.ac.uk.

\section{Notes}

The authors declare no competing financial interest.

\section{ACKNOWLEDGMENTS}

S.Y. and A.M.E. wish to thank the U. K. Engineering and Physical Science Research Council (EPSRC) and the Leverhulme Trust for grants in support of this work and the Advanced Microscopy Centre at the University of Leicester for providing TEM facilities.

\section{REFERENCES}

(1) Hu, J.; Odom, T. W.; Lieber, C. M. Acc. Chem. Res. 1999, 32, $435-445$.

(2) Morales, A. M.; Lieber, C. M. Science 1998, 279, 208-211.

(3) Wang, Z. L. Adv. Mater. 2000, 12, 1295-1298.

(4) Liu, X.-Y.; Liu, M.-H.; Luo, Y. C.; Mou, C.-Y.; Lin, S. D.; Cheng, H.-K.; Chen, J.-M.; Lee, J.-F.; Lin, T.-S. J. Am. Chem. Soc. 2012, 134, 10251-10258.

(5) Xia, Y. N.; Yang, P. D.; Sun, Y. G.; Wu, Y. Y.; Mayers, B.; Gates, B.; Yin, Y. D.; Kim, F.; Yan, Y. Q. Adv. Mater. 2003, 15, 353-389.

(6) Van Houten, H.; Beenakker, C. Phys. Today 1996, 49, 22-27.

(7) Dreher, M.; Pauly, F.; Heurich, J.; Cuevas, J. C.; Scheer, E.; Nielaba, P. Phys. Rev. B 2005, 72, 075435.

(8) Pascual, J. I.; Méndez, J.; Gómez-Herrero, J.; Baró, A. M.; Garcia, N.; Landman, U.; Luedtke, W. D.; Bogachek, E. N.; Cheng, H.-P. Science 1995, 267, 1793-1795.

(9) Fagas, G.; Greer, J. C. Nano Lett. 2009, 9, 1856-1860.

(10) Zhu, G.; Zhang, S.; Xu, Z.; Ma, J.; Shen, X. J. Am. Chem. Soc. 2011, 133, 15605-15612.

(11) Arutyunov, K. Y. Phys. C (Amsterdam, Neth.) 2008, 468, 272275.

(12) Dresselhaus, M. S.; Chen, G.; Tang, M. Y.; Yang, R. G.; Lee, H.; Wang, D. Z.; Ren, Z. F.; Fleurial, J.-P.; Gogna, P. Adv. Mater. 2007, 19, $1043-1053$

(13) Zeng, X. Q.; Latimer, M. L.; Xiao, Z. L.; Panuganti, S.; Welp, U.; Kwok, W. K.; Xu, T. Nano Lett. 2011, 11, 262-268.

(14) Jain, A.; Nair, P. R.; Alam, M. A. Proc. Natl. Acad. Sci. U. S. A. 2012, 109, 9304-9308.

(15) Li, H. H.; Zhao, S.; Going, M.; Cui, C. H.; He, D.; Liang, H. W.; Wu, L.; Yu, S. H. Angew. Chem., Int. Ed. 2013, 52, 7472-7276.

(16) Lieber, C. M. MRS Bull. 2003, 28, 486-491.

(17) Hong, B. H.; Bae, S. C.; Lee, C.-W.; Jeong, S.; Kim, K. S. Science 2001, 294, 348-351.
(18) Malisauskas, M.; Meskys, R.; Morozova-Roche, L. A. Biotechnol. Prog. 2008, 24, 1166-1170.

(19) Liu, Z. P.; Xu, D.; Liang, J. B.; Shen, J. M.; Zhang, S. Y.; Qian, Y. T. J. Phys. Chem. B 2005, 109, 10699-10704.

(20) Teng, X. W.; Han, W. Q.; Ku, W.; Hücker, M. Angew. Chem., Int. Ed. 2008, 47, 2055-2058.

(21) Cademartiri, L.; Ozin, G. A. Adv. Mater. 2009, 21, 1013-1020.

(22) Liu, R.; Liu, J. F.; Jiang, G. B. Chem. Commun. 2010, 46, 70107012.

(23) Yang, S. C.; Hong, F.; Wang, L. Q.; Guo, S. W.; Song, X. P.; Ding, B. J.; Yang, Z. M. J. Phys. Chem. C 2010, 114, 203-207.

(24) Hong, X.; Wang, D. S.; Yu, R.; Yan, H.; Sun, Y.; He, L.; Niu, Z. Q.; Peng, Q.; Li, Y. D. Chem. Commun. 2011, 47, 5160-5162.

(25) Koenigsmann, C.; Santulli, A. C.; Gong, K. P.; Vukmirovic, M. B.; Zhou, W. P.; Sutter, E.; Wong, S. S.; Adzic, R. R. J. Am. Chem. Soc. 2011, 133, 9783-9795.

(26) Zhang, W.-H.; Zhang, W.-D. Mater. Lett. 2013, 98, 5-7.

(27) Xia, B.; Wu, H.; Yan, Y.; Lou, X.; Wang, X. J. Am. Chem. Soc. 2013, 135, 9480-9485.

(28) Toennies, J. P.; Vilesov, A. F. Angew. Chem., Int. Ed. 2004, 43, 2622-2648.

(29) Yang, S.; Ellis, A. M.; Spence, D.; Feng, C.; Boatwright, A.; Latimer, E.; Binns, C. Nanoscale 2013, 5, 11545-11553.

(30) Mozhayskiy, V.; Slipchenko, M. N.; Adamchuk, V. K.; Vilesov, A. F. J. Chem. Phys. 2007, 127, 094701.

(31) Loginov, E.; Gomez, L. F.; Vilesov, A. F. J. Phys. Chem. A 2011, $115,7199-7204$.

(32) Boatwright, A.; Cheng, F.; Spence, D.; Latimer, E.; Binns, C.; Ellis, A. M.; Yang, S. Faraday Discuss. 2013, 162, 113-124.

(33) Volk, A.; Thaler, P.; Koch, M.; Fisslthaler, E.; Grogger, W.; Ernst, W. E. J. Chem. Phys. 2013, 138, 214312.

(34) Donnelly, R. J. Quantized Vortices in Helium II; Cambridge University Press: Cambridge, England, 1991.

(35) Bewley, G. P.; Lathrop, D. P.; Sreenivasan, K. R. Nature 2006, $441,588$.

(36) Bewley, G. P.; Paoletti, M. S.; Sreenivasan, K. R.; Lathrop, D. P. Proc. Natl. Acad. Sci. U. S. A. 2008, 105, 13707-13710.

(37) Paoletti, M. S.; Fiorito, R. B.; Sreenivasan, K. R.; Lathrop, D. P. J. Phys. Soc. Jpn. 2008, 77, 111007.

(38) Gordon, E. B.; Karabulin, A. V.; Matyushenko, V. I.; Sizov, V. D.; Khodos, I. I. Chem. Phys. Lett. 2012, 519-520, 64-68.

(39) Lebedev, V.; Moroshkin, P.; Grobety, B.; Gordon, E.; Weis, A. J. Low Temp. Phys. 2011, 165, 166-176.

(40) Lehmann, K. K.; Schmied, R. Phys. Rev. B 2003, 68, 224520.

(41) Dalfovo, F.; Mayol, R.; Pi, M.; Barranco, M. Phys. Rev. Lett. 2000, 85, 1028-1031.

(42) Grisenti, R. E.; Toennies, J. P. Phys. Rev. Lett. 2003, 90, 234501.

(43) Gomez, L. F.; Loginov, E.; Vilesov, A. F. Phys. Rev. Lett. 2012, $108,155302$.

(44) Spence, D.; Latimer, E.; Cheng, C.; Boatwright, A.; Ellis, A. M.; Yang, S. Phys. Chem. Chem. Phys. 2014, 16, 6903-6906.

(45) Gomez, L. F.; Loginov, E.; Sliter, R.; Vilesov, A. F. J. Chem. Phys. 2011, 135, 154201.

(46) Loginov, E.; Gomez, L. F.; Chiang, N.; Halder, A.; Guggemos, N.; Kresin, V. V.; Vilesov, A. F. Phys. Rev. Lett. 2011, 106, 233401.

(47) Witten, T. A., Jr; Sander, L. M. Phys. Rev. Lett. 1981, 47, 14001403.

(48) Liang, C. L.; Zhong, K.; Liu, M.; Jiang, L.; Liu, S. K.; Xing, D. D.; Li, H. Y.; Na, Y.; Zhao, W. X.; Tong, Y. X.; Liu, P. Nano-Micro Lett. 2010, 2, 6-10. 